\title{
In-operando soft X-ray microspectroscopy of organic electronics devices
}

Rainer H. Fink ${ }^{1,6}$, Benedikt Rösner ${ }^{1,2}$, Xiaoyan Du ${ }^{1,3}$, Andreas Späth ${ }^{1}$, Manuel Johnson ${ }^{1}$, Tim Hawly ${ }^{1}$, Benjamin Watts ${ }^{4}$, Jörg Raabe ${ }^{4}$, Luca Gregoratti ${ }^{5}$, Matteo Amati ${ }^{5}$

${ }^{1 .}$ Physical Chemistry II, Friedrich-Alexander-Universität Erlangen-Nürnberg (FAU), Erlangen, Germany.

2. present address: LMN, Paul Scherrer Institut, Villigen, Switzerland

3. present address: i-MEET, Friedrich-Alexander-Universität Erlangen-Nürnberg, Erlangen, Germany

4. Swiss Light Source, Paul Scherrer Institut, Villigen, Switzerland

${ }^{5 .}$ Elettra-Sincrotrone Trieste, Area Science Park, Trieste, Italy

6. CENEM, Friedrich-Alexander-Universität Erlangen-Nürnberg, Erlangen, Germany.

* Corresponding author, rainer.fink@fau.de

Organic semiconducting films, e.g., pentacene films, bear high potential for organic electronics such as organic field-effect transistors (OFETs). Charge transport in thin organic films is a crucial issue for pushing the performance to state-of-the-art levels [1]. However, its exact mechanism still remains one of the key challenges for future investigations [2]. The charge carrier mobility is often strongly influenced by the microstructure of the active film. Its microstructural order has hence to be carefully considered for the engineering of high-performance devices [3]. Although there are several studies on the characterization of OFETs under operation, the vast majority of reports deals with morphology and spectroscopy separately. For instance, scanning transmission X-ray microscopy (STXM), atomic force microscopy (AFM), grazing incidence X-ray diffraction or Raman spectroscopy enable to study the granular microstructure of pentacene films. On the other hand, Raman spectroscopy, near-edge X-ray absorption fine structure (NEXAFS) or sum frequency generation have disclosed average spectroscopic changes induced by transistor operation [4]. However, most of these spectroscopic studies are methodically limited to average across the electrically active semiconductor film, which means that they are not capable of probing the changes locally. Apart from scanning Kelvin probe microscopy (SKPM) [3], the impact of charges on the local electronic structures remains widely unexplored.

The complementary use of STXM imaging, Raman microspectroscopy and Scanning Photoemission microscopy (SPEM) allows us to obtain more detailed insight into the interplay of thin-film OFET morphology, charge transport and modification of the electronic structure in operating devices. The microscopic tools allow to probing the properties within the active channel of the OFET while changing the transport characteristics of the devices, i.e., in the on- and off-states. In the present cases, STXM imaging based on NEXAFS dichroism offers access to the domain structure within the organic film (see Fig. 1) and modifications in the unoccupied density of states (UDOS). SPEM in contrast probes the occupied (core and valence) levels to evaluate molecular polarization effects at the organic-dielectric interface. Raman microspectroscopy on the contrary uses local polarizability and is thus dependent on local charges.

We have used different organic semiconductors for the present studies: pentacene as prototype p-type semiconductor with sufficiently high charge-carrier mobility, PDI- $\mathrm{FCN}_{2}$ and $\alpha, \omega$-dihexyl-quarterthiophene. In all cases, we can correlate the charge-transport with the polycrystalline film morphology due to charge trapping at the grain boundaries. The gate effect induces variations in the UDOS due to energetic level shifts and thus partial filling/depletion of the LUMO levels. Since both occupied and unoccupied states are affected in the on-state of the OFET, energetic shifts are not accessible from 
NEXAFS alone. We therefore utilized the knowledge of SPEM-XPS to judge on the gate effects.

To reduce the effect of grain boundaries we have improved a novel technique to overcome drawbacks of conventional, i.e., vacuum-sublimated organic thin films [5] Using molecular self-organization at the liquid-liquid interface and subsequent transfer onto solid substrates, we are able to prepare extended single-domain crystalline films with extensions of about several square millimeters an thicknesses of few molecular layers only. The reduction of structural defects like grain boundaries leads to a significant increase (factor 5 - 10) in the charge carrier mobilities in thus prepared top-contact/bottom-gate fieldeffect transistors. Such devices will therefore offer advances in molecular electronics in terms of performance and structural definition.

With the present study the complementary use of x-ray based spectroscopic nanoprobes sheds new light on energy level shifts in organic electronic devices beyond conventional probes like e.g. Kelvin probe force microscopy [6].

[1] M. Halik et al., Adv. Mater., 15, 2003, 917.

[2] G. Witte, C. Wöll, J. Mater. Res., 19, 2004, 1889.

[3] M. Tello et al., Adv. Funct. Mater., 18, 2008, 3907.

[4] B. Rösner et al., Organic Electronics, 15, 2014, 435.

[5] C. Xu et al., Angew. Chem. (Int. Ed). 55, 2016, 9519

[6] M. Nakamura et al., App. Phys. Lett. 86, 2005, 122112

[7] The authors gratefully acknowledge funding by the German Minister of Education and Research (BMBF, contract 05 K16 WED) and the Deutsche Forschungsgemeinschaft within GRK 1896. X.D. obtained additional support through the China Scholarship Council (CSC). We would like to thank Dr. N. Pillet for experimental support.

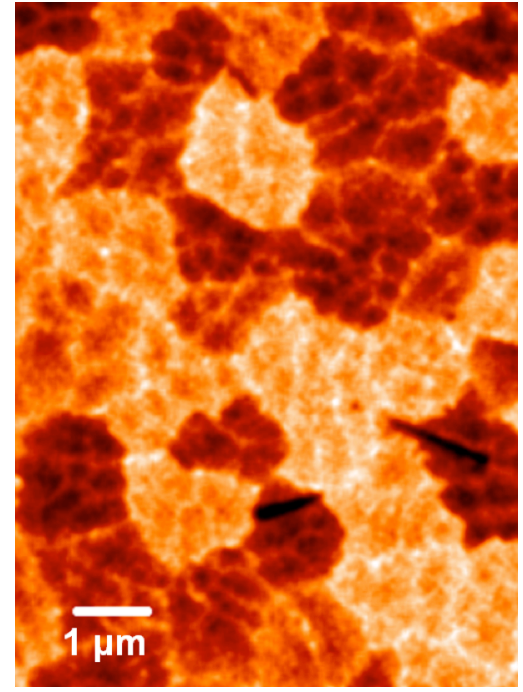

Figure. 1. STXM micrograph of pentacene (30 $\mathrm{nm}$ thick). Contrast is due to azimuthally rotated microcrystalline domains (FOV: $6 \times 9 \mathrm{~m}^{2}$ ).

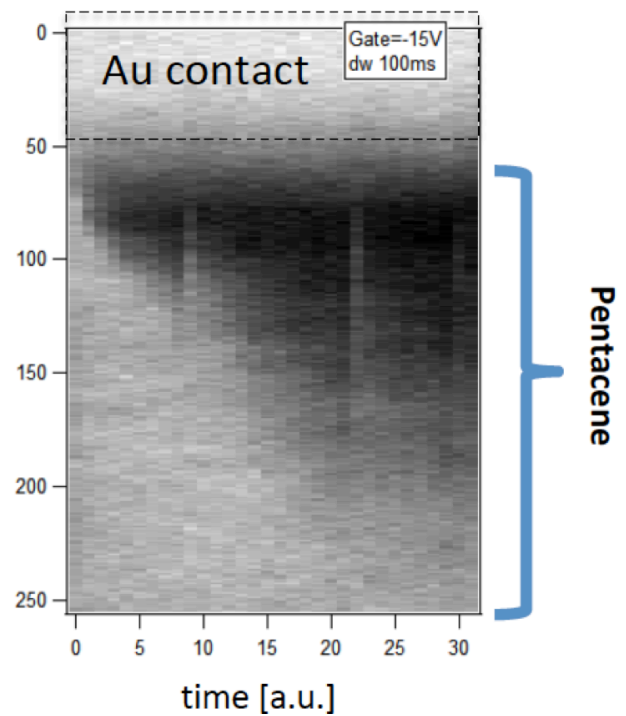

Figure. 2. C 1s SPEM micrograph within the active channel of a pentacene OFET. Contrast is due to charge accumulation in the channel as derived from $\mathrm{C} 1 \mathrm{~s}$ shifts with increasing $\mathrm{X}-$ ray illumination times. 